\title{
Toxina Botulínica para el Tratamiento de los Desórdenes Temporomandibulares
}

\author{
Botulinum Toxin for the Treatment of Temporomandibular Disorders
}

\author{
Natalia Alvarez-Pinzon'; Eder Alberto Sigua-Rodriguez²,3; Douglas Rangel Goulart ${ }^{4}$; \\ Sergio Olate ${ }^{5,6}$; Gustavo Jacobucci Farah ${ }^{3}$ \& Liogi Iwaki Filho ${ }^{3}$
}

\begin{abstract}
ALVAREZ-PINZON, N.; SIGUA-RODRIGUEZ, E. A.; GOULART, D. R.; OLATE, S.; FARAH, G. J.; IWAKI FILHO, L. Toxina botulínica para el tratamiento de los desórdenes temporomandibulares. Int. J. Odontostomat., 12(2):103-109, 2018.

RESUMEN: Los Trastornos Temporomandibulares (TTM) consisten en un conjunto de condiciones patológicas que afectan la articulación temporomandibular (ATM), los músculos de la masticación y las estructuras asociadas. Estudios epidemiológicos estiman que $40 \%$ a $75 \%$ de la población adulta presenta por lo menos un signo de TTM, como ruido articular y $33 \%$ por lo menos un síntoma, como dolor facial o en la ATM. La toxina botulínica (BoNT) es una neurotoxina producida por la bacteria anaerobia Clostridium botulinum, solo la toxina A y B son utilizadas en la práctica clínica después de la aprobación de la Food and Drug Administration en 1989 y 2000; actualmente continúan siendo utilizadas para tratar varias condiciones de dolor, incluyendo la espasticidad muscular, distonia, dolor de cabeza y dolor miofascial. Las propuestas del mecanismo de acción fueron sugeridas a mediados de 1950 manifestando que esta neurotoxina posee alta afinidad con la sinapsis colinérgicas, ocasionando un bloqueo en la liberación de acetilcolina de esos terminales nerviosos, sin alterar la conducción neural de las señales eléctricas o en la síntesis de almacenamiento de acetilcolina. La inyección intramuscular en dosis y localización apropiada, provoca denervación química parcial y disminución de la contractura, sin ocasionar parálisis completa a lo que se le atribuye ser un innovador y eficaz método de tratamiento para el dolor crónico asociada con hiperactividad de los músculos masticatorios. La toxina botulínica tipo A es ser una alternativa para el control de la sintomatología dolorosa presente en los TTMs de etiología miogénica. Los autores recomiendan realizar un correcto diagnóstico, ya que la indicación de este tipo de tratamiento con diagnósticos incorrectos lleva a resultados inciertos.
\end{abstract}

PALABRAS CLAVE: toxina botulínica, trastorno temporomandibular, dolor crónico.

\section{INTRODUCCIÓN}

Los Trastornos Temporomandibulares (TTM), es un término colectivo usado para describir un conjunto de condiciones que incluye la articulación temporomandibular (ATM), los músculos de la masticación y estructuras asociadas. En Norte América la incidencia de esta condición es cerca del $10 \%$, dentro de los síntomas más comunes se encuentra la sensibilidad en los músculos de la masticación, el ruido articular, el desvío en la trayectoria de abertura bucal y algunos pacientes pueden presentar dolor irradiado en la región auricular (Freund et al., 2000).
La toxina botulinica (BoNT) es una neurotoxina producida por la bacteria anaerobia Clostridium botulinum y es considerada como una de las toxinas más potentes, fue desarrollada en 1981 como medicamento y aplicado en los músculos de los ojos para el tratamiento del estrabismo, después de diversas pruebas en laboratorios fue aprobada por la Food And Drug Administration (FDA) para ser empleada en varios tratamientos de disturbios de movimiento manifestados como anormal, excesiva o inapropiada contracción y aumento del tono muscular (Sposito, 2009).

\footnotetext{
${ }^{1}$ Práctica Privada, Bogotá, Colombia.

${ }^{2}$ Centro de Investigaciones del Colegio Odontológico (CICO), Institución Universitaria Colegios de Colombia, Bogotá, Colombia.

${ }^{3}$ Department of Dentistry, Oral, and Maxillofacial Surgery, State University of Maringá, Maringá, Paraná, Brazil.

${ }^{4}$ Oral and Maxillofacial Surgery, Centro Universitário Euro-Americano - UNIEURO, Brasília, Brazil.

${ }^{5}$ División de Cirugia Oral, Facial y Maxilofacial, Universidad de La Frontera, Temuco, Temuco, Chile.

${ }^{6}$ Centro de Excelencia en Estudios Morfológicos y Quirurgicos (CEMyQ), Universidad de La Frontera, Temuco, Chile.
} 
El mecanismo de acción más conocido, consiste en la inhibición de la liberación de acetilcolina en los terminales nerviosos motores, llevando a una disminución de la contracción muscular lo cual permite su aplicación clínica y terapéuticamente en enfermedades donde existe exceso de la contracción muscular (Dressler et al., 2005; Sposito).

La BoNT/A es una toxina biológica muy potente; actualmente estan descritas siete neurotoxinas distintas, todas con diferentes potencias, duración de efecto y actúan en diferentes proteínas intracelulares. Solo la toxina A y B son utilizadas en la práctica clínica después de la aprobación de la Food and Drug Administration (FDA) en 1989 y 2000; actualmente continuan siendo utilizadas para tratar varias condiciones de dolor, incluyendo la espasticidad muscular, distonia, dolor de cabeza y dolor miofascial (Colhado et al., 2009).

La BoNT también tiene un efecto antinocioceptivo mediante el bloqueo de la liberación de mediadores inflamatorios, tales como la sustancia $P$ y glutamato; debido a su posible efecto analgésico y relajante muscular, ha ganado mucho interés como opción de tratamiento en dolores neuropáticos, dolor miofascial y dolor de cabeza (Ernberg et al., 2011). El objetivo de esta revisión es mostrar el uso de Toxina Botulinica en el control de la sintomatología dolorosa de los desórdenes temporomandibulares.

\section{TRASTORNOS TEMPORO MANDIBULARES (TTM)}

El síndrome de dolor miofascial es una condición regional que implica dolor y rigidez muscular, caracterizada por la presencia de puntos gatillos miofascial (PG). Clínicamente, esos PG son bandas tensas hipersensibles focales que producen una respuesta de contracción local y clasico patrón de dolor local referida a la palpación (Ho \& Tan, 2007).

Mense (2004) afirmó que la formación de PG se inicia con una lesión del músculo (por ejemplo sobrecarga) que lleva a una liberación excesiva de acetilcolina de la unión neuromuscular, la acetilcolina provoca despolarización de la membrana sublimiar del músculo postsináptico que es seguido por la liberación de $\mathrm{Ca}++$ a partir de almacenamientos intracelulares, las del retículo sarcoplasmatico, así el aumento de la concentración de Ca++ lleva al deslizamiento de los filamentos de actina y miosina que forman la contractura local (Mense). La etiología de los TTMs es multifactorial incluyendo aspectos psicosociales y sobrecarga de los músculos de la masticación y estructuras músculo-esqueléticas relacionadas a la cabeza y cuello (Dall'Antonia et al., 2013).

Estudios epidemiológicos estiman que $40 \%$ a 75 $\%$ de la población adulta presenta por lo menos un signo de TTM, como ruido articular y $33 \%$, por lo menos un síntoma, como dolor en la cara o en la articulación. Ernberg et al. relataron que la prevalencia de TTM llega a ser $5-10 \%$ más alta en mujeres que en hombres y se han propuesto varias posibilidades para explicar esas diferencias, en términos de diferencias psicosociales, hormonales y constitucionales, sin resultados concluyentes (Poveda-Roda et al., 2007).

Las causas de estos síntomas son diversas e incluyen trauma, iatrogenia, alteraciones oclusales, estrés y algunos desequilibrios emocionales. La salud mental juega un papel dominante en la patogénesis de los TTMs; por otro lado, el sistema neuromuscular responsable de la función masticatoria tiene un alto potencial de adaptación a condiciones que agreden este complejo sistema, pero cuando las capacidades compensatorias del sistema masticatorio y el sistema neuromuscular son sobrecargados se produce la disfunción que resulta en síntomas como dolor, ruidos articulares y/o movimientos mandibulares limitados, lo que obliga al paciente a buscar tratamiento (de Figueiredo et al., 2009; Dall'Antonia et al.; Wieckiewicz et al., 2015).

Daif (2012) indicó que la hipertonía de los músculos de la masticación es considerada una de la causas del dolor relacionado a TTM, que generalmente resulta de la hiperactividad muscular masticatoria e hipermovilidad que tiende a irradiarse en la región de los músculos afectados tanto en reposo como después del ejercicio físico excesivo, de forma que los músculos más afectados son los elevadores de la mandíbula (músculos masetero, temporal y pterigoideo medial) y los proyectores de la mandíbula (músculo pterigoideo lateral) (von Lindern, 2001).

El dolor muscular es transmitido por fibras aferentes nerviosas de grupo III y IV para el sistema nervioso central que procesa la cantidad, intensidad, duración y localización del estímulo nocivo. El uso en exceso de una musculatura por movimientos repetitivos ocasiona traumatismos que generan contracción muscular localizada y liberación de sustancias algógenas promoviendo dolor local, esta alteración muscular provoca aumento de la liberación de acetilcolina y una exacerbada crisis de energía es perpetuada dentro de la banda tensa muscular (Dall'Antonia et al.). 
En estas condiciones, terapias conservadoras son indicadas como el tratamiento de elección, al menos en la primera fase, entre las cuales están: placas de desoclusión, terapias físicas, fármacos (analgésicos y relajantes musculares) auto-masaje, ajustes oclusales y educación del paciente (Freund et al.).

Las placas oclusales tienen importancia dentro de las terapias conservadoras y se utlizan con este objetivo desde hace mas de 100 años (Conti et al., 2012). La terapia con placa mio-relajante ofrece la posibilidad de llegar a oclusión en relación céntrica, eliminación de las interferencias oclusales, aumento de la dimensión vertical, reducción de la actividad neuromuscular y establecimiento de relaciones oclusales estables con contactos dentarios uniformes en todo el arco dentario (Michelotti et al., 2012); debido a su eficacia es comúnmente utilizada en experimentos para el grupo control.

Aproximadamente el $80 \%$ de los pacientes puede obtener buenos resultados con estas terapias; sin embargo, cuando los síntomas de dolor son de tipo crónico, en el $20 \%$ de los pacientes no van a ser totalmente utiles estas terapias (Brin, 2009). La BoNT comenzó a ser una alternativa en el control de la sintomatología dolorosa asociada a la hiperactividad muscular, presentando una elección promisoria a los tratamientos tradicionales (GuardaNardini et al., 2008).

\section{MECANISMO DE ACCIÓN DE LA BoNT}

La BoNT consiste en una combinación compleja de proteínas que contiene neurotoxina botulínica y varias proteínas no toxicas; está constituida por una cadena pesada y una cadena leve unidos por un único enlace di-sulfuro (Dressler et al.). Fue considerada letal por muchos siglos, siendo que sus síntomas clínicos y musculares fueron descritos con detalles sólo en el inicio del siglo XIX por el médico Justinus Kerner (Dall'Antonia et al.).

Existen siete serotipos diferentes denominadas A, B, C, D, E, F y G. A pesar de todos estos serotipos inhibir la liberación de acetilcolina de las terminaciones nerviosas, sus proteínas diana intracelular, su acción y sus características varían sustancialmente. En la actualidad solo se emplea el serotipo A por ser el más ampliamente estudiado con fines terapéuticos y ocasionalmente, el tipo B (Dall'Antonia et al.; GarciaRuiz, 2013).
La BoNT está disponible comercialmente en cuatro marcas distintas, tres de tipo A (Botox®, Dysport $₫$ y Xeomin $®$ ) y una marca tipo $B$ (Neurobloc $®$ ) (Dressler et al.; Garcia-Ruiz). Las dosis no son relacionadas debido a las diferencias en el proceso de formulación (Mooney et al., 2003).

BoNT/A es un agente biológico obtenido en laboratorio, siendo una sustancia cristalina estable, liofilizada, asociada a la albumina humana y utilizada después de la dilución en solución fisiológica y medida en unidades biológicas $(U)$ definidas por la dosis letal 50 (DL50) (Unno et al., 2005).

Las propuestas del mecanismo de acción fueron sugeridas a mediados de 1950 (Dall'Antonia et al.) manifestando que esta neurotoxina posee alta afinidad con la sinapsis colinérgicas, ocasionando un bloqueo en la liberación de acetilcolina de esos terminales nerviosos, sin alterar la conducción neural de los señales eléctricos o en la síntesis de almacenamiento de acetilcolina. La inyección intramuscular en dosis y localización apropiada, provoca denervación química parcial y disminución de la contractura, sin ocasionar parálisis completa (Clark, 2003; Ho \& Tan; Colhado et al.) a lo que se le atribuye ser un innovador y eficaz método de tratamiento para el dolor crónico asociada con hiperactividad de los músculos masticatorios (von Lindern).

Se cree que también tiene un efecto antinocioceptivo bloqueando la liberación de mediadores inflamatorios como la sustancia $\mathrm{P}$ y el glutamato, lo que sugiere que ese alivio de dolor no sería simplemente gracias al relajamiento muscular que produce más también a través de la acción a nivel central, inhibiendo la liberación de mediadores del dolor (Ernberg et al.).

Otro local de acción analgésica puede ser la terminación nerviosa simpática postganglionar que usa noradrenalina y ATP como neurotransmisores. La norepinefrina es conocida para aumentar los casos de dolor crónico y el ATP es un estimulante de nociceptores musculares; si la BoNT/A inhibe la liberación de estos transmisores, puede ser analgésico en caso de dolor mantenida simpáticamente incluyendo el síndrome de dolor regional compleja (Mense).

\section{PROTOCOLO DE APLICACIÓN DE LA BONT/A PARA EL TRATAMIENTO DE LA TTM DE ORIGEN MIOGENICO}

Las dosis y técnicas de aplicación utilizadas para 
el control del dolor muscular y síntomas propios de los TTM han sido diferentes en los experimentos citados en la literatura. Para determinar la cantidad de fármaco a ser administrado inicialmente debemos medir el volumen de los músculos temporal y masetero bilateralmente. En músculos de gran volumen utilizamos dosis mayores distribuidos de la siguiente manera: en el músculo temporal $25 \mathrm{U}$ en cada lado, divididos en 5 puntos de aplicación y en el masetero $50 \mathrm{U}$ divido en 5 puntos de aplicación (Fig. 1). Por otro lado en músculos de menor volumen disminuimos la dosis para $10 \mathrm{U}$ en el temporal en cada lado y 30 en el masetero, es importante citar que para el tratamiento de dolor miogenico relacionado a TTM la aplicación deberá ser realizada con las mismas dosis bilateralmente.

La posición del paciente es sentado con el plano oclusal paralelo al piso; se realiza la antisepsia con gluconato de clorhexidina al $2 \%$ en la región temporal y maseterica (Fig. 2) y con la aplicación de anestésico tópico para piel (Fig. 3) se espera por 25 minutos para obtener el efecto deseado (según indicación del fabricante). Posteriormente realizamos la aplicación en la región anterior del musculo temporal posicionando el conjunto aguja-jeringa con una inclinación aproximadamente de 45 grados con respecto al músculo (Fig. 4) y realizamos la aplicación lentamente. Una vez aplicada en el temporal, realizamos la aplicación en los músculos maseteros, en este caso se deberá posicionar el conjunto aguja-jeringa perpendicular al musculo (Fig. 5). La aguja que utilizamos es de $30 \mathrm{G}$ o $32 \mathrm{G}$ con $13 \mathrm{~mm}$ de largo con una jeringa luer look de tuberculina de $1 \mathrm{~mL}$.

Como recomendaciones inmediatas sugerimos que durante las cuatro primeras horas no se debe realizar ningún tipo de actividad física, evitar movimientos en los músculos faciales y de la masticación (sonreír, cantar, masticar, hablar) que generen posibles migraciones del fármaco a estructuras no deseadas, no masajear la zona de aplicación, realizar aplicación de hielo y durante las primeras semanas mantener una dieta blanda.

\section{APLICACIONES CLÍNICAS}

La distribución local y sistémica de BoNT depende de los siguientes factores: (1) propagación, que se refiere a un movimiento físico de la toxina de un sitio a otro y que depende de un número de variables relacionadas con la técnica de inyección, el volumen, tamaño de la aguja, y otros factores físicos;

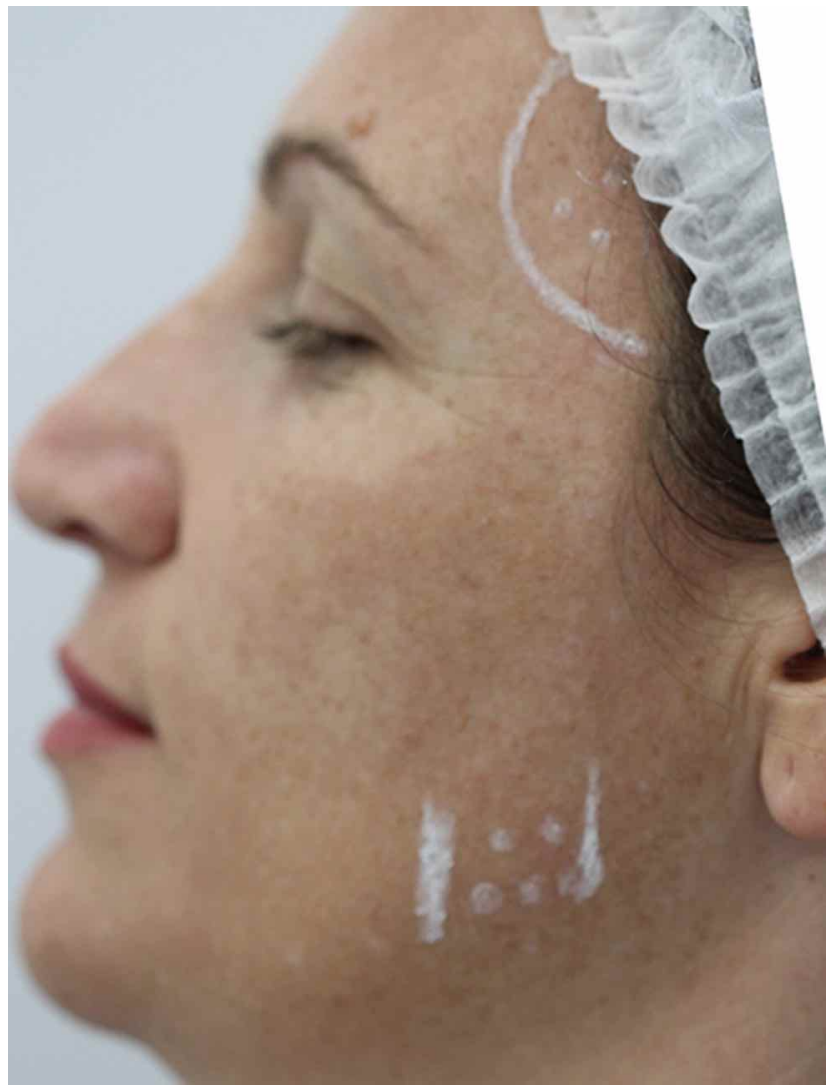

Fig. 1. Marcación con lápiz blanco mostrando el área y puntos de aplicación, el color evita tatuajes indeseados.

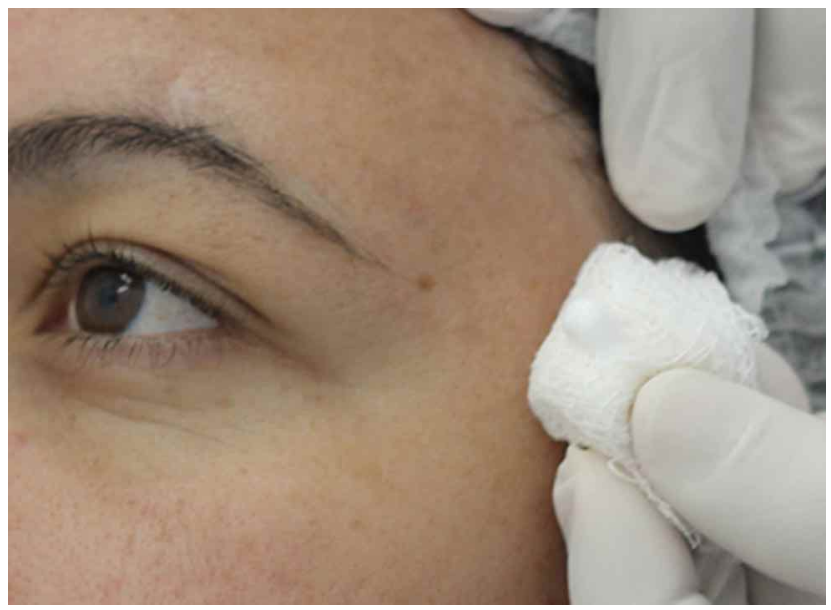

Fig. 2. Antisepsia con gluconato de clorhexidina al $2 \%$ en la región que será aplicada la Toxina Botulínica.

(2) difusión, que se refiere a un fenómeno scopic, más micro en la que una molécula soluble se dispersa por un transporte pasivo más allá de su sitio de la inyección inicial; (3) migración, que se refiere a la propagación para sitios distantes que pueden ocurrir ya sea a través de los nervios (transporte axonal 


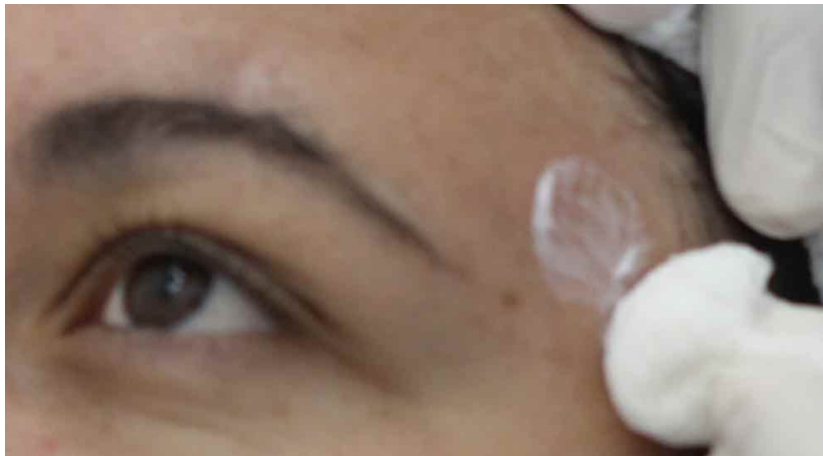

Fig. 3. Aplicación de anestésico tópico de piel tipo EMLA (lidocaína $25 \mathrm{mg} / \mathrm{g}$ y prilocaína $25 \mathrm{mg} / \mathrm{g}$ ) en región temporal y masetérica.

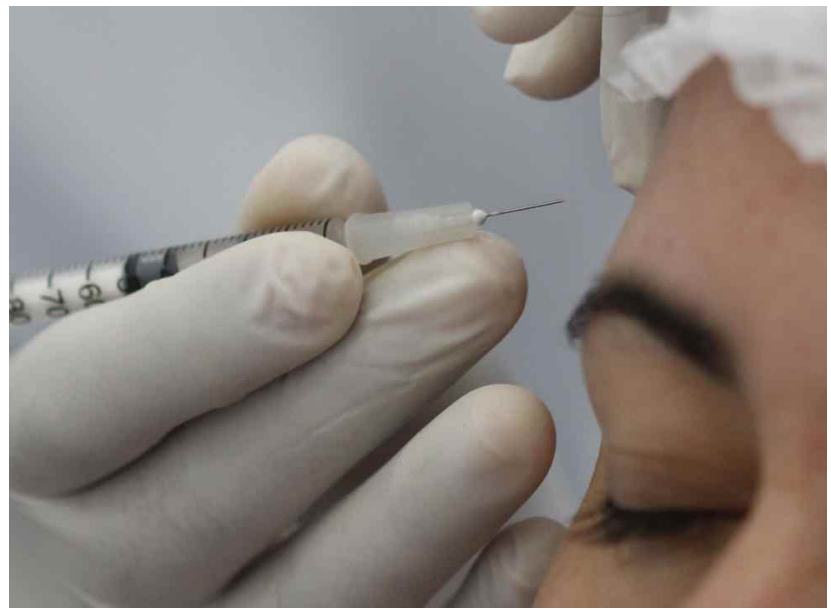

Fig. 4. Palpación y aplicación en el musculo temporal con la aguja inclinada aproximadamente a $45^{\circ}$ con respecto al músculo.

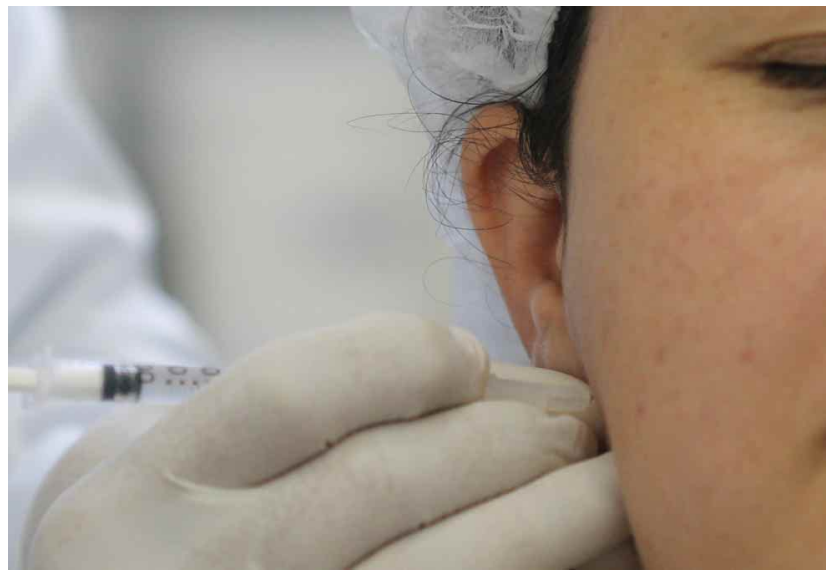

Fig. 5. Aplicación de la Toxina Botulínica tipo A en el músculo masetero, notar que la aguja debe estar perpendicular al musculo como lo muestra la imagen.

neuronal) o por la sangre (transporte hematógena) y (4) el volumen y dilución (Ramirez-Castaneda et al., 2013).
Von Lidern (2001) realizó un ensayo clínico controlado, randomizado y ciego con 90 pacientes que presentaban dolor crónico como resultado de la hiperactividad de los músculos de la masticación más específicamente los músculos elevadores de la mandibula; el dolor fue medido por medio de la escala visual analógica (EVA) antes y después del tratamiento, en el que a los 90 pacientes sometidos al estudio, 60 recibieron aplicaciones de BoNT/A (Botox ${ }^{\circledR}$, Allergan, Ettlingen Germany) en dosis de $35 \mathrm{U}$ diluida en $0,7 \mathrm{~mL}$ de solución salina, inyectadas bilateralmente en los músculos temporal, masetero y pterigoideo medial y en los 30 pacientes restantes fueron inyectados con $0,7 \mathrm{~mL}$ de solución salina como grupo control, el tiempo de estudio fue de 12 semanas; los resultados de este estudio mostró que hubo una mejoría de los síntomas de dolor local en 55 casos $(91 \%)$ para el grupo tratado con BoNT/A.

Freund et al. realizaron un estudio con 46 pacientes de ambos géneros, con síntomas de dolor muscular y artrogénica, a los cuales se le aplicaron $50 \mathrm{U}$ de BoNT/A en los músculos maseteros y $25 \mathrm{U}$ en los músculos temporal, en 5 lugares diferentes de aplicación bilateral para cada músculo, segun orientación establecida en la electromiografía. Los individuos fueron evaluados en intervalos de dos semanas, durante 8 semanas, utilizando la escala visual analógica (EVA), la medición de la contracción máxima voluntaria (CMV), la abertura bucal interincisivos, la sensibilidad a la palpación y un índice funcional con múltiplos valores de EVA. Después de la inyección ocurrió una reducción del dolor subjetiva según en EVA en 40 de los 46 pacientes ( $87 \%$ ) y una reducción de dolor objetiva (sensibilidad a la palpación) en 44 de los 46 pacientes (96\%).

La BoNT/A no solamente es usada para el control de tipo miogenica, también para disfunción de tipo articular. Fueron reportados dos casos de uso de toxina botulínica para el tratamiento de desplazamiento anterior del disco, donde las aplicaciones fueron realizadas en el músculo pterigoideo lateral; se utilizó BoNT/A (Botox®; Allergan, Irvine Califórnia) reconstituida con solución salina estéril al $0,9 \%$ en una concentración de $10 \mathrm{U}$ por $0,1 \mathrm{~mL}$ y fueron utilizadas $30 \mathrm{U}$ para la inyección en cada pterigoideo lateral bilateralmente. El músculo fue abordado de intraoral y lateralmente a la tuberosidad de la maxila, en el medio de su origen e inserción, se introdujo una aguja larga sobre los molares superiores paralelos al plano oclusal y para la correcta ubicación de la aguja fue necesario el uso de la electromiografía. El procedi- 
miento fue repetido dos veces cada tres meses con un intervalo de seis meses entre cada aplicación. Se obtuvo como resultado la eliminación total del ruido articular durante el periodo de observación de un año (Bakke et al., 2005).

Se ha observado que la cantidad y la concentración óptima de BoNT/A para producir analgesia y relajamiento de la fibra muscular inflamada son factores importantes para que sus aplicaciones alcancen el efecto deseado, aunque la falta de un protocolo definido impide saber cuál es la dosis correcta de la BoNT/A para reducir la sintomatología dolorosa de los TTMs.

Ho \& Tan en el (2007), realizaron una revisión sistemática con el objetivo de evaluar la eficacia de la BoNT/ A en comparación con placebo inyectada en puntos gatillo para el dolor miofascial. Cinco ensayos clínicos cumplieron los criterios de inclusión; un estudio concluyó que la BoNT/ A fue eficaz y cuatro mostraron que la toxina botulínica no era capaz de reducir el dolor decurrente de los puntos gatillos para el dolor miofacial. Ho \& Tan concluyeron que la toxina botulínica no tiene mayor eficacia cuando es comparada con otras terapias como inyección con suero fisiológico, anestésicos locales o agujamiento seco; indicaron ademas que el costo de la toxina botulínica es alta y nuevos estudios serian necesaior para establecer su relación costo-beneficio.

Por otro lado, Schawartz \& Freud en el (2002), mostraron resultados favorables con dosis de $25-50 \mathrm{U}$ para el musculo masetero y 5-25U para el músculo temporal, que fueron consideradas dosis seguras reportando mínimos efectos adversos; igualmente de su eficacia, el refinamiento de un cierto número de parámetros con respecto a la aplicación puede potencializar los efectos de la BoNT/A (Kim et al., 2003), principalmente relacionado con la localización de punción y dosis del medicamento (Freund et al.).

En otro estudio realizado por Kurtoglu et al. (2008), fueron evaluados los efectos de la BoNT/A en pacientes con dolor miofascial con o sin desplazamiento de disco de la ATM. La muestra fue veinticuatro pacientes divididos en dos grupos; en un grupo se aplicaron BoNT/A en dosis de $30 \mathrm{U}$ en los músculos maseteros divididos en tres pontos y $20 \mathrm{U}$ en el músculo temporal, divididos en dos puntos aplicados bilateralmente. En el grupo control aplicaron solución salina igual en los músculos temporal y maseteros, después de las inyecciones los resulta- dos mostraron una mejoría en el dolor de los pacientes tratados con BoNT/A (Kurtoglu et al.).

A pesar de las controversias, la dosis recomendada para el músculo masetero en su porción superficial y profunda es de 40-60U por musculo, inyectadas en dos o tres lugares de la porción superficial; para el músculo temporal porciones anterior, media y posterior, se recomienda de 30-50U por músculo, inyectadas en cuatro lugares en la porción anterior, media y posterior, la dosis no debe ultrapasar 200U por músculo (Clark). La dosis letal en seres humanos no es conocida, aunque ha sido estimada cerca de $3.000 \mathrm{U}$ (Katz, 2005).

Estudios preliminares muestran que la toxina botulínica ha sido utilizada con éxito para tratar varios síndromes de dolor incluyendo los TTM; la naturaleza compleja de los TTM y la proximidad de los músculos con al nervio facial, la técnica de inyección correcta y orientaciones de dosis adecuadas son muy importantes para un resultado exitoso (Schwartz \& Freund, 2002).

En base a la información obtenida, podemos indicar que la toxina botulínica tipo A es una alternativa para el control de la sintomatología dolorosa presente en los TTMs de etiología miogénica. Los autores recomiendan realizar un correcto diagnóstico, ya que la indicación de este tipo de tratamiento con diagnósticos incorrectos lleva a resultados inciertos, creando falsos resultados. Probablemente hoy, el uso de la toxina botulinaca no es la primera opción de tratamiento para el dolor miofascial, pero es una alternativa útil asociada a otros tratamientos cuando las medidas conservadoras no producen resultados satisfactorios.

ALVAREZ-PINZON, N.; SIGUA-RODRIGUEZ, E. A.; GOULART, D. R.; OLATE, S.; FARAH, G. J.; IWAKI FILHO, L. Botulinum Toxin for the Treatment of Temporomandibular Disorders. Int. J. Odontostomatol.,12(2):103-109, 2018.

ABSTRACT: Temporomandibular Disorders (TMD) is a term that was used to describe a set of pathological conditions that affect temporomandibular joint (TMJ), muscles of mastication and associated structures. Epidemiological studies estimates that $40 \%$ to $75 \%$ of the adult population has at least one sign of TMD, such as joint noise and $33 \%$ presented some symptom such as facial or joint pain. Botulinum toxin is a neurotoxin produced by the anaerobic bacterium Clostridium botulinum. There are two types of toxin ( $A$ and $B$ ) used in clinical practice that were approved by Food and Drug Administration in 1989 and 2000. These 
medications are in use to treat various diseases including muscle spasticity, dystonia, headache and myofascial pain. The mechanisms of action were suggested in the mid-1950s, this neurotoxin seems to have an action at cholinergic synapses, causing a block in the release of acetylcholine from the nerve terminals without altering the neural conduction of the electrical signals. The synthesis and storage of acetylcholine were preserved. Intramuscular injection in appropriate doses and location causes partial chemical denervation and decreased contracture, without causing complete paralysis of muscles. Due to this features, it has been considered an innovative and effective method to treat chronic pain associated with hyperactivity of masticatory muscles. The botulinum toxin type $A$ appears to be an alternative method for pain control present in TMDs that have myogenic etiology. The authors recommend a correct diagnosis, since the indication of this type of treatment associated with an incorrect diagnosis leads to uncertain outcomes, creating false conclusions.

KEY WORDS: botulinum toxins, temporomandibular disorder, chronic pain.

\section{REFERENCIAS BIBLIOGRÁFICAS}

Bakke, M.; Møller, E.; Werdelin, L. M.; Dalager, T.; Kitai, N. \& Kreiborg, S. Treatment of severe temporomandibular joint clicking with botulinum toxin in the lateral pterygoid muscle in two cases of anterior disc displacement. Oral Surg. Oral Med. Oral Pathol. Oral Radiol. Endod., 100(6):693-700, 2005.

Brin, M. F. Basic and clinical aspects of BOTOX. Toxicon, 54(5):67682, 2009.

Clark, G. T. The management of oromandibular motor disorders and facial spasms with injections of botulinum toxin. Phys. Med. Rehabil. Clin. N. Am., 14(4):727-48, 2003.

Colhado, O. C.; Boeing, M. \& Ortega, L. B. Botulinum toxin in pain treatment. Rev. Bras. Anestesiol., 59(3):366-81, 2009.

Conti, P. C.; de Alencar, E. N.; da Mota Corrêa, A. S.; Lauris, J. R.; Porporatti, A. L. \& Costa, Y. M. Behavioural changes and occlusal splints are effective in the management of masticatory myofascial pain: a short-term evaluation. J. Oral Rehabil., 39(10):754-60, 2012.

Daif, E. T. Correlation of splint therapy outcome with the electromyography of masticatory muscles in temporomandibular disorder with myofascial pain. Acta Odontol. Scand., 70(1):72-7, 2012.

Dall'Antonia, M.; de Oliveira Netto, R. M.; Sanches, M. L. \& Guimarães, A. S. Jaw muscles myofascial pain and botulinum toxin. Rev. Dor., 14(1):52-7, 2013.

de Figueiredo, V. M. G.; Cavalcanti, A. L.; de Farias, A. B. L. \& do Nascimento, S. R. Prevalência de sinais, sintomas e fatores associados em portadores de disfunção temporomandibular. Acta Sci. Health Sci., 31(2):159-63, 2009.

Dressler, D.; Saberi, F. A. \& Barbosa, E. R. Botulinum toxin: mechanisms of action. Arq. Neuropsiquiatr., 63(1):180-5, 2005.

Ernberg, M.; Hedenberg-Magnusson, B.; List, T. \& Svensson, P. Efficacy of botulinum toxin type $A$ for treatment of persistent myofascial TMD pain: a randomized, controlled, double-blind multicenter study. Pain, 152(9):1988-96, 2011.

Freund, B.; Schwartz, M. \& Symington, J. M. Botulinum toxin: new treatment for temporomandibular disorders. Br. J. Oral Maxillofac. Surg., 38(5):466-71, 2000.
Garcia-Ruiz, P. J. Aplicaciones de la toxina botulínica en Neurología. Med. Clin., 141(1):33-6, 2013.

Guarda-Nardini, L.; Manfredini, D.; Salamone, M.; Salmaso, L.; Tonello, S. \& Ferronato, G. Efficacy of botulinum toxin in treating myofascial pain in bruxers: a controlled placebo pilot study. Cranio, 26(2):12635, 2008.

Ho, K. Y. \& Tan, K. H. Botulinum toxin A for myofascial trigger point injection: a qualitative systematic review. Eur. J. Pain, 11(5):51927, 2007.

Katz, H. Botulinum toxins in dentistry--the new paradigm for masticatory muscle hypertonicity. Singapore Dent. J., 27(1):7-12, 2005.

Kim, H. S.; Hwang, J. H.; Jeong, S. T.; Lee, Y. T.; Lee, P. K.; Suh, Y. L. \& Shim, J. S. Effect of muscle activity and botulinum toxin dilution volume on muscle paralysis. Dev. Med. Child. Neurol., 45(3):2006, 2003.

Kurtoglu, C.; Gur, O. H.; Kurkcu, M.; Sertdemir, Y.; Guler-Uysal, F. \& Uysal, H. Effect of botulinum toxin-A in myofascial pain patients with or without functional disc displacement. J. Oral Maxillofac. Surg., 66(8):1644-51, 2008.

Mense, $\mathrm{S}$. Neurobiological basis for the use of botulinum toxin in pain therapy. J. Neurol., 251 Suppl. 1:11-7, 2004.

Michelotti, A.; lodice, G.; Vollaro, S.; Steenks, M. H. \& Farella, M. Evaluation of the short-term effectiveness of education versus an occlusal splint for the treatment of myofascial pain of the jaw muscles. J. Am. Dent. Assoc., 143(1):47-53, 2012.

Poveda-Roda, R.; Bagan, J. V.; Díaz Fernández, J. M.; Hernández Bazán, S. \& Jiménez Soriano, Y. Review of temporomandibular joint pathology. Part I: classification, epidemiology and risk factors. Med. Oral Patol. Oral Cir. Bucal, 12(4):E292-8, 2007.

Ramirez-Castaneda, J.; Jankovic, J.; Comella, C.; Dashtipour, K.; Fernandez, H. H. \& Mari, Z. Diffusion, spread, and migration of botulinum toxin. Mov. Disord., 28(13):1775-83, 2013.

Schwartz, M. \& Freund, B. Treatment of temporomandibular disorders with botulinum toxin. Clin. J. Pain, 18(6 Suppl.):S198-203, 2002.

Sposito, M. M. M. Toxina Botulínica do Tipo A: mecanismo de ação. Acta Fisiatr., 16(1):25-37, 2009.

Unno, E. K.; Sakata, R. K. \& Issy, A. M. Comparative study between botulin toxin and bupivacaine for triggering-points infiltration in chronic myofascial syndrome. Rev. Bras. Anestesiol., 55(2):250$5,2005$.

von Lindern, J. J. Type A botulinum toxin in the treatment of chronic facial pain associated with temporo-mandibular dysfunction. Acta Neurol. Belg., 101(1):39-41, 2001.

Wieckiewicz, M.; Boening, K.; Wiland, P.; Shiau, Y. Y. \& ParadowskaStolarz, A. Reported concepts for the treatment modalities and pain management of temporomandibular disorders. J. Headache Pain, 16:106, 2015.

Dirección para correspondencia:

Eder Alberto Sigua-Rodriguez

Department of Dentistry, Oral and Maxillofacial Surgery

State University of Maringá

Maringá, Paraná

BRAZIL

Email: edersiguaodont@gmail.com

Recibido : 11-02-2018

Aceptado: 13-04-2018 